\title{
Phytochemical analysis and antibacterial activities of some plant extracts on Staphylococcus aureus isolates from patients receiving hospital treatments in Ekiti State, Nigeria
}

\author{
Marshanty Obem Oyama ${ }^{1}$, Adeola Oluwagbemileke Egbebi ${ }^{1}$, Fred Coolborn Akharaiyi ${ }^{2 * \mathbb{D}}$ \\ ${ }^{1}$ Biological Sciences, Afe Babalola University, Ado Ekiti, Ekiti State, Nigeria \\ ${ }^{2}$ Microbiology Department, Edo University Iyamho, Edo State, Nigeria
}

\section{A R T I C L E I N F O}

Article Type:

Original Article

\section{Article History:}

Received: 20 June 2018

Accepted: 5 December 2018

\section{Keywords:}

Plant extract

Herbal medicine

Medicinal plant

Staphylococcus aureus

Nigeria

\begin{abstract}
A B S T R A C T
Introduction: Resistance of Staphylococcus aureus to available antibiotics is in alarming rate to put this into control with the use of natural products of plant derivatives. The aim of this research is centered on comparative study of plant extracts and antibiotics on S. aureus isolates from hospital patients.

Methods: A total of 106 human clinical samples were collected and analyzed for S. aureus isolates from urine, noses, ears and wounds of patients. The isolated $S$. aureus species were subjected to inhibition with plant extracts in comparison with commercial antibiotics. Twenty S. aureus isolates from the subjects' samples were analyzed for multidrug resistance to antibiotics and plant leaves aqueous extracts. Out of these isolates, 9 were obtained from urine, 3 from noses, and 4 each from ears and wounds samples. These isolates were identified with code numbers Results: The highest inhibition created on the isolates with modern medicine was between 11 and $35 \mathrm{~mm}$ while for traditional medicine inhibition of the isolates was between 11 and $36 \mathrm{~mm}$. Alkaloids and saponins were more than others in the plants extracts where value of alkaloids was between 2.42 and 3.63 and saponins between 2.60 and 3.27 .

Conclusion: The plants extract inhibition was comparable to the antibacterial potency of commercial antibiotics on the tested bacteria species. The antibacterial activities observed from Terminalia catappa, Mangifera indica and Acalypha wilkesiana are probably due to several bioactive compounds contained in the plants and may serve as templates alternative medicine to treat infections caused by $S$. aureus.
\end{abstract}

Implication for health policy/practice/research/medical education:

The study confirms the antibacterial activities of Terminalia catappa, Mangifera indica and Acalypha wilkesiana on the isolated $S$. aureus species. The several bioactive compounds contained in these plants extracts may serve as templates alternative medicine to treat infections caused by $S$. aureus.

Please cite this paper as: Oyama MO, Egbebi AO, Akharaiyi FC. Phytochemical analysis and antibacterial activities of some plant extracts on Staphylococcus aureus isolates from patients receiving hospital treatments in Ekiti State, Nigeria. J Herbmed Pharmacol. 2018;9(1):14-20. doi: 10.15171/jhp.2019.03.

\section{Introduction}

Staphylococcus aureus is a world widely threatening bacteria pathogen. Its infections are very severe if not treated on time and will gradually progress to manifest certain disorders such as infections of the tissue, wounds, bone, joint and pneumonia (1). Treatment of S. aureus infections remain very challenging because in recent years Staphylococcus has been discovered to resist the popular antibiotics (tetracycline, methicillin, gentamicin [GEN], lincosamides and macrolides) used for treatment. Methicillin-resistant Staphylococcus aureus (MRSA) has been implicated as a serious problem in many medical institutions for its life-threatening situations (2). Also, the existence of mutated strains of MRSA is the vancomycin resistant $S$. aureus (VRSA) that has contributed additional problems to health care communities. The VRSA is at present the most threat of $S$. aureus strain to humans hence vancomycin has been misused for the treatment of Staphylococci infections (2).

Majority of data on prevalence of S. aureus in Africa are patient-based. Despite this, the 'one health' concept, including environmental persistence should be a vital 
consideration for the effective control measure of this organism. Many studies in Nigeria have the information that in the hospital environment, infections due to $S$. aureus is the most prevalent (50\%-80\%) (3). In the neonatal intensive care units in Ghana, infections due to S. aureus accounts for $44 \%$ (4). S. aureus association on door handles and bathtubs in hospital environment in Benin implicated the Panton-Valentine leukocidin (PVL) as cause of nosocomial diseases (5).

The biological scientist communities have been attracted recently of the use of medicinal plants. The isolation and identification of effective secondary metabolites from these plants made them useful for herbal remedies.

In health care system in Nigeria, diseases are treated with plants of medicinal values in conjunction with modern medicine. Folklore is believed to be based on holistic approach to good health, thus it perceives success and misfortune depending on the actions of individual and ancestral spirits on the balance or imbalance in the gap of the individual and social environment. This is however, in contrast to modern medicine that is based on technicality and analyses.

The aim of this research is centered on phytochemical screening of plant extracts and comparative studies of plants extracts and antibiotics on $S$. aureus isolated from hospital patients.

\section{Materials and Methods}

\section{Sample collection}

A total of 106 human clinical samples were collected and analyzed for $S$. aureus isolation. The samples were collected from urine, wound, nose and ear of both male and female patients of different ages hospitalized in different wards of Ekiti State University Teaching Hospital (EKSUTH), the State specialist hospital, Ikere-Ekiti and General Hospital Iyin-Ekiti in Ekiti State Nigeria. From men, 33 urine samples, 14 ear swabs, 4 nose swabs and 7 wound swabs were obtained. On the other hand, a total of 45 samples were obtained from women comprising 25 urine samples, ten ear swabs 6 nose swabs and 7 wound swabs.

Each urine sample was aseptically collected into a sterile sampling bottle, while ear, nose and wound swabs were collected using a sterile swab stick.

\section{Plant Samples}

Fresh plant leaves of Terminalia catappa (Almond), Mangifera indica (Mango) and Acalypha wilkesiana (Acalypha) were obtained within the premises of Afe Babalola University and identified by Mr. Esimekhuai Donatus a botanist in the Department of Botany University of Ibadan Nigeria. Voucher specimens for T. catappa (UIH 22567), M. indica (UIH 22568) and A. wilkesiana (UIH 22569) were deposited at the herbarium of the Department of Botany, University of Ibadan, Nigeria. The leaves were brought to the laboratory, stripped from their stems, washed with distilled water and macerated into a fine infusion using a laboratory mechanical grinder. The solution was filtered with No. 1 Whatman filter paper and finally passed through membrane filter to obtain sterile extract. These were labeled and kept at $4^{\circ} \mathrm{C}$ in the refrigerator until use.

\section{Phytochemical screening}

Qualitative analysis

To detect the presence of qualitative bioactive constituents from the plants extracts, standard chemical methods were employed with the criteria of Harborne (6) and Chethana et al (7).

\section{Quantitative screening}

Quantitative phytochemical screening (flavonoids, tannins, saponins total phenol and alkaloids) from the plants extracts was done by the methods previously described by Harborne (6) and Chethana et al (7).

\section{Antibiotic susceptibility test}

Inoculums were prepared from saline suspension of isolates cultured for 18 to 24 hours. McFarland solution was used to adjust the solution to match the 0.5 standard of McFarland turbidity. With use of sterile swabs, inoculums were obtained from the adjusted suspensions which were allowed to drain properly before streaking onto freshly prepared Mueller-Hinton agar. The streaking was spread all over the surface of culture medium by rotating the plates at $60^{\circ} \mathrm{C}$ for even distribution of inoculums. The antibiotics disc was placed on the agar surface and pressed firmly with sterile forceps. The inoculated plates were left at room temperature for 15 minutes before they were incubated for $24-48$ hours at $35^{\circ} \mathrm{C}$. Effectiveness of the antibiotics was shown by clear zones around them which are measured and interpreted in accordance to the criteria of Clinical and Laboratory Standards Institute, as whether the pathogen is resistant, intermediate or sensitive (8).

\section{Plants extract inhibitory test}

The plants extracts were evaluated for antimicrobial potency by well-in-agar diffusion method. The surface of freshly prepared plates of Mueller-Hinton agar was spread plated with the bacterial inoculums and allowed to stand for 2 hours. Then, the wells of $6 \mathrm{~mm}$ were aseptically made with a sterile cork borer on the seeded agar plates. A volume of $50 \mu \mathrm{L}$ from each extract solution was filled into the wells. The inoculated agar plates were incubated at $37^{\circ} \mathrm{C}$ for 24 to 48 hours. Clear inhibition zones after incubation were measured with a transparent ruler and interpreted as the degree of sensitivity.

Statistical analysis

The statistical tool used for testing of hypothesis was 
the analysis of variance (ANOVA). Data generated were expressed as mean \pm standard error mean (SEM) and tested through one-way ANOVA or by students $t$ test (8). $P<0.05$ was considered significant.

\section{Results}

Susceptibility of isolates to antibiotics

Twenty $S$. aureus isolates from the subjects' samples were analyzed for multidrug resistance to antibiotics and the plant leaves aqueous extracts. Out of these isolates, 9 were obtained from urine, 3 from noses, 4 each from ears and wounds of patients. These isolates were identified with code numbers Ui-Uiii and ESi-Esiii, respectively for urine and ear isolates.

Twenty of $S$. aureus isolates from the clinical samples were all susceptible to ofloxacin (OFL) with zones of inhibition that ranged from 25 to $35 \mathrm{~mm}$, followed by GEN with inhibition zone of between $16-23 \mathrm{~mm}$ and erythromycin (ERY) with zones between 11 and $25 \mathrm{~mm}$. All the isolates were resistant to ceftazidime (CAZ) and cloxacillin (CXC). Nine of the isolates were sensitive to cefuroxime (CRX) with zones of inhibition ranged from 10 to $25 \mathrm{~mm}$. Eleven of the isolates were sensitive to CTR with inhibition zones between 11 and $20 \mathrm{~mm}$. Meanwhile only one isolate was sensitive to augmentin (AUG) and was inhibited with a zone of $20 \mathrm{~mm}$ (Figure 1).

Susceptibility of isolates to plant extracts

The sensitivity pattern of the $S$. aureus isolates to leaves extract is represented in Figure 2. T. catappa exhibited the highest level of effectiveness against 18 isolates from urine, ear, nose and wounds with inhibition zones between 16 and $36 \mathrm{~mm}$. Only 2 isolates were resistant to this plant extract. Nineteen isolates were inhibited with $M$. indica leaf extract with zones ranged from 11 to $32 \mathrm{~mm}$ inhibition. Fourteen out of the 20 isolates were resistant to the aqueous leaf extract of A. wilkesiana while 6 of the isolates were susceptible with inhibitory zones that ranged from 11 to $20 \mathrm{~mm}$.

Phytochemical constituents

Table 1 presents the qualitative phytochemicals present in the leaves aqueous extracts of the plants. Tannin was more abundant in T. catappa than $M$. indica and A. wilkesiana. Phenol content was higher in T. catappa than others. Reducing sugars were present in all the leaf extracts. They were more in A. wilkesiana than other leaf extracts. Terpenoids were absent in T. catappa and $M$. indica but were more abundant in A. wilkesiana. Steroids and flavonoids were present in all plant extracts. Cardiac glycosides were present in both T. catappa and $M$. indica but totally absent in A. wilkesiana. Glycosides were abundantly present in T. catappa and A. wilkesiana but moderately present in $M$. indica.

The quantity of tannins present in the plants extracts was $0.04 \pm 0.01,0.06 \pm 0.01$ and $0.03 \pm 0.01$ for T. catappa, A. wilkesiana and $M$. indica, respectively.

Flavonoids content was $0.17 \pm 0.03,0.17 \pm 0.02$ and $0.00 \pm 0.00$ in T. catappa, M. indica and A. wilkesiana respectively. No significant difference existed between the phenol quantities in T. catappa, $(0.03 \pm 0.01), M$. indica $(0.00 \pm 0.03)$ and A. wilkesiana (0.04 \pm 0.02$)$. Also, the quantity of saponins in T. catappa $(2.60 \pm 0.02)$ was not significantly different from the values of $M$. indica $(3.31 \pm 0.36)$ and A. wilkesiana (3.27 \pm 0.01$)$. Alkaloids values from T. catappa, M. indica and A. wilkesiana were $3.63 \pm 0.01,2.42 \pm 0.60$ and $3.52 \pm 0.01$, respectively (Table 2 ).

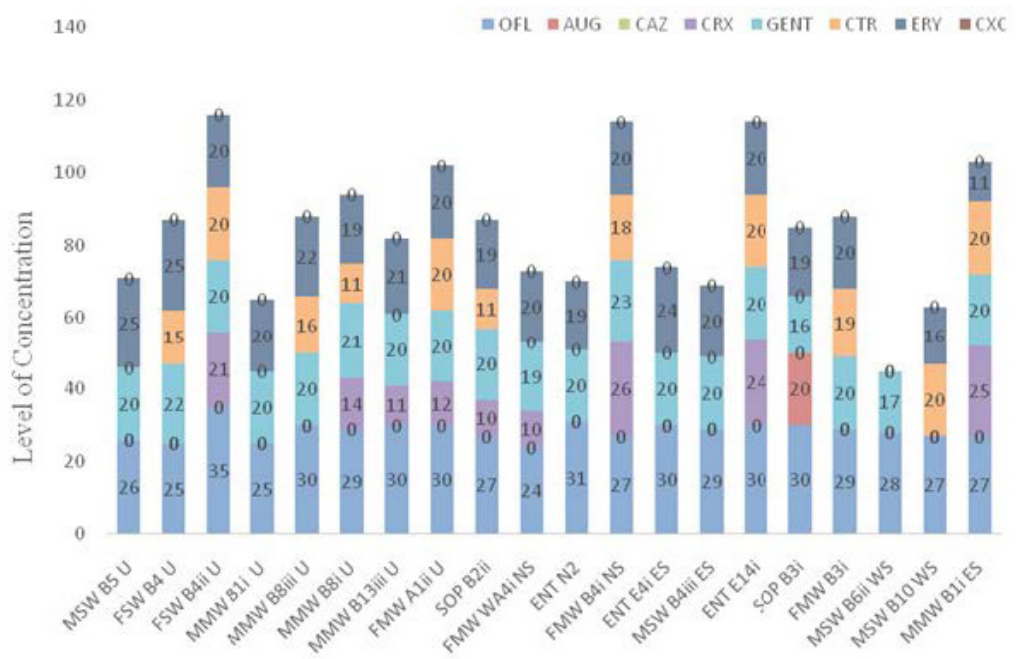

Figure 1. Susceptibility pattern of Staphylococcus aureus to antibiotics.

MSW, male surgical ward; FSW, female surgical ward; MMW, male medical ward; FMW, female medical ward; SOP, surgical out patient; ENT, ear nose and throat, B, A, W (5, 4,1, 8, 3, 6, 10 respectively), Bed no.; ES= ear swab, NS, nose swab; WS, wound swab. (ofloxacin, augmentin, ceftazidime, cefuroxime, gentamycin, ceftriaxone, erythromycin and cloxacillin). 


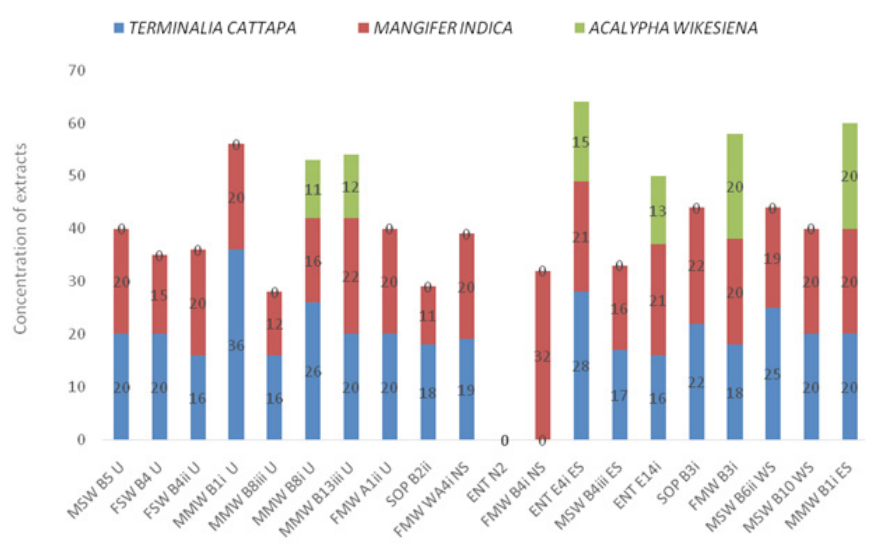

Figure 2. Inhibition of Staphylococcus aureus isolate with plants extracts.

Key: $\mathrm{MSW}=$ male surgical ward, $\mathrm{FSW}=$ female surgical ward, $\mathrm{MMW}=$ male medical ward, $\mathrm{FMW}=$ female medical ward, $\mathrm{SOP}=\mathrm{surgical}$ out patient, ENT = ear nose and throat, $B, A, W(5,4,1,8,3,6,10$, respectively $)=$ Bed no., $E S=$ ear swab, NS=nose swab and $W S=$ wound swab.

\section{Discussion}

The commercial antibiotics studied revealed that OFL was the most effective antibiotic on $S$. aureus isolates. This apparently level of susceptibility to OFL suggest that it could be a preferred drug for treating $S$. aureus infections in the area studied for this research, especially at the present time. This finding is consistent with previous reports $(16 ; 17)$. However, low sensitivity was reported among most strains of MRSA isolated from patients with ocular infections (9).

$S$. aureus susceptibility to Gentamycin in present study compares favorably with reports published in the Southwestern part of Nigeria (10). Gentamycin is supposed to be an alternative antibiotic for treating infections of MRSA.

In the past years, CAZ has basically been prescribed for treating Staphylococcal infections because of dependable sensitivity results it presented in vitro. This was reported by Ndip et al. (11) with $78 \%$ and Amadi et al (12) who recorded sensitivity of $85.4 \%$. Meanwhile, the susceptibility of $S$. aureus to cloxacillin (CXC) as observed in this study is in variance with the current trend. However, there is

Table 1. Qualitative phytochemicals from the aqueous leaf extracts

\begin{tabular}{lccc}
\hline $\begin{array}{l}\text { Phytochemical } \\
\text { constituents }\end{array}$ & T. catappa & M. indica & A. wilkesiana \\
\hline Alkaloids & ++ & ++ & ++ \\
Tannin & +++ & ++ & ++ \\
Phenol & +++ & ++ & ++ \\
Saponin & + & + & + \\
Terpenoids & - & - & +++ \\
Steroids & +++ & ++ & + \\
Flavonoids & +++ & +++ & + \\
Cardiac glycosides & + & + & - \\
Reducing sugars & ++ & ++ & +++ \\
Glycosides & + & ++ & + \\
\hline
\end{tabular}

+++: Abundantly present, ++: Moderately present, +: Present, -: Absent. no correlation with the reports of some other researchers (13) where they all observed low susceptibility response to CXC. The resistance in high-level could be associated with earlier exposure of these antibiotics to bacteria which may have developed resistance hence the level of antibiotic abuse is relatively high in this area of study. This could arise from self-medication, inadequate completion of prescribed drugs and inadequate prescription of drugs (14). Complete resistance of the S. aureus isolates to CAZ was observed. This could be due to over use of this drug by individuals and such could develop resistance to this drug overtime. However, this finding is contrasting to the observation of Dang-Thic et al (15) who reported total susceptibility with CAZ. Dang-Thic et al (15) stated that the current market has many products containing ceftiofur (a third-generation cephalosporin) as such pharmacists should be concerned about the development of resistance to this antibiotic in the near future.

While 9 of the isolates were sensitive to CRX, only one was sensitive to AUG. The observed low resistance to CRX and AUG belonging to the fluoroquinolones, and the susceptibility of all isolated $S$. aureus species to OFL and GEN is in support of the previous findings by Oguzkaya-Artan et al (16). The profound activity of these agents on bacteria species may obviously be as a result of mode of inhibition which is principally bactericidal. The low resistance of isolated $S$. aureus to AUG could result from the presence of a compound (clavulanic acid) which is a competitive inhibitor of beta-lactamases. This compound restores the antimicrobial potency of beta-lactam antibiotics against $S$. aureus. S. aureus is a bacterium known for its ability to secret beta-lactamase. $S$. aureus infections can effectively be treated with CRX, a second-generation cephalosporin agent which has ability to resist beta-lactamases. These antibiotics which showed varied activities on the isolated $S$. aureus species could be as a statement of their expensiveness and therefore cannot 
Oyama et al

Table 2. Quantitative phytochemicals from the aqueous leaf extract

\begin{tabular}{llllll}
\hline Samples & Tannin & Flavonoids & Phenol & Saponin \\
\hline T. catappa & $0.38 \pm 0.87^{\mathrm{a}}$ & $0.17 \pm 0.03^{\mathrm{a}}$ & $0.03 \pm 0.06$ & $2.60 \pm 0.02$ & $3.63 \pm 0.87^{\mathrm{a}}$ \\
M. indica & $0.28 \pm 0.05^{\mathrm{b}}$ & $0.17 \pm 0.02^{\mathrm{a}}$ & $0.33 \pm 0.03$ & $3.31 \pm 0.36$ & $3.42 \pm 0.60$ \\
A. wilkesiana & $0.56 \pm 0.07^{\mathrm{a}}$ & $0.00 \pm 0.00$ & $0.04 \pm 0.02$ & $3.27 \pm 0.87$ & $3.52 \pm 0.87^{\mathrm{b}}$ \\
S. V. $(\alpha=0.05)$ & $(P=0.02)$ & 0.00 & 0.87 & 0.96 \\
\hline
\end{tabular}

Values are mean \pm standard deviations of triplicate determinations. Values with different superscripts per column are significantly different.

easily be misused. The availability of this antibiotic in injectable form which requires administration by experts is also a factor.

The high susceptibility rate of the $S$. aureus isolates to ERY was contrasting to the findings of Onanuga et al who observed a moderately high resistance (17). The resistance effect could have occurred as a result of the drugs uncontrolled usage, due to selection pressure that favors the increase of resistant strains.

It has been reported in surveys that almost all introduced antibiotics have encountered resistance by microorganisms (18). Outside that, some antibiotics have presented severe side effects on hosts, where depletion of beneficial gut and mucosal microorganisms, hypersensitivity, immunosuppression and allergic reactions are inclusive (19). The recorded inhibition effect with the employed standard reference antibiotic compares with the plants extract potency on the isolated S. aureus species. Aqueous extract of $A$. wilkesiana leaves demonstrated the same level of inhibition as AUG. Furthermore, this leaf extract expressed a greater rate of antimicrobial potency on $S$. aureus isolates compared to CAZ and CXC. However, Oluduro et al (20) reported that S. aureus and S. typhi developed resistance to amoxicillin. In their study, these bacteria species were inhibited with aqueous, ethanol and methanol leaf extracts. The highest antimicrobial activity was observed with T. catappa which was of better effect on 18 isolates with wider zones of inhibition than the commercial antibiotics used in this study. Also, aqueous leaf extract of $M$. indica demonstrated higher antimicrobial effect on 19 isolates than the antibiotics used except OFL from which a wider zone of inhibition was observed. This is an indication that the crude leaf extracts were as effective as the employed commercial antibiotics. The effectiveness of the plants extracts on the isolated $S$. aureus species confirms their use against $S$. aureus-mediated infections and also gastroenteritis and skin dermatitis. Since remarkable inhibition was observed on the isolated $S$. aureus species, the exhibited antibacterial activity might be of broad spectrum potential (20).

All the $S$. aureus isolates from urine, ear and wound samples responded with very high levels of susceptibility to the aqueous extracts of T. catappa and $M$. indica leaves. However, 6 isolates ( 3 from urine and 3 from ear samples) showed susceptibility to A. wilkesiana. On the other hand, the aqueous leaf extract of $A$. wilkesiana was not effective against $S$. aureus isolates of nose and wound samples. A. wilkesiana Muell Arg extract among other Acalypha species, has been found useful for its medicinal value in treating malaria fever, fungal infections and gastrointestinal disorder (21), antihypertension properties (22) and for its antimicrobial activities (23). The reason for its non-inhibitory effect to $S$. aureus isolated from nose and wound samples are not clear. Also, neither $A$. wilkesiana nor T. catappa and M. indica inhibited S. aureus isolated from nose samples. The presence of terpenoids in the aqueous extract of $A$. wilkesiana leaves corresponds with the reports from previous studies by Oluduro et a. (20). A report by Awe and Eme (24) suggested that due to antibacterial activities of A. wilkesiana, in inhibition of both Gram positive bacteria species (S. aureus) and Gram negative bacteria species (Klebsiella pneumoniae, Shigella sp. and E. coli), it could be a broad spectrum antibacterial agent. The phytochemical compounds screened from the plant leaves determined their antibacterial potency. Meanwhile, some of chemicals such as flavonoids, saponins and tannins have been reported in several literatures as antibacterial agents. The qualitative and quantity phytochemicals screened from the leaves of $A$. wilkesiana showed the presence of useful antibacterial plant chemicals (flavonoid, saponin, reducing sugars, terpenoids and tannin) in varying proportions and also with traces of glycosides and steroids. These could be attributed to the antimicrobial activities observed with this plant extract.

The phytochemical screening showed the presence of some bioactive components of alkaloids, tannin, saponin, steroids, cardiac glycosides, flavonoids, reducing sugars, glycosides, triterpenoids and phenol in all plant extracts. Antimicrobial activities have been displayed by many plants with high contents of phenols and alkaloids compounds. The quantities of saponin present in the aqueous extract of the 3 leaves extracts were not statistically significant.

The high susceptibility of isolates to the aqueous extract of $M$. indica leaves may be attributed to the bioactive constituents present in this plant. These compounds contained in $M$. indica could be a good source of potential bioactive compounds for the treatment of infectious diseases such as pneumonia as reported by Ncube et al (25). M. indica leaf extract has antibacterial affinity against E. coli and other bacteria species under Enterobacteriaceae family. In the report of Khan et al. (26), the potential pharmacology associated with mangiferin inclusive of 
its antimicrobial and antioxidant activities has been identified. Also, its in vitro effect on type II $5 a$-reductase, gastroprotective and antidiabetic effect in rodents have been reported (26). From results obtained in this study, it can be deduced that aqueous extract of T. catappa leaves is one of the very best sources of treatment for infections as extract of this plant leaves demonstrated the highest level of microbial inhibition against all $S$. aureus species isolated from patient's urine, ear, wound and nose samples. This finding supports the reports of other researchers who used different solvents in the extraction of the plant leaves. T. catappa leaves are rich in tannin flavonoids, alkaloids and steroids. These components are able to inhibit certain phenotypic expression of quorum sensing (QS) in some test strains (27). In a study of antibacterial activity of T. catappa aqueous leaves extract in different stages extracted with water against various harmful microorganisms, the results showed that T. catappa leaves had the capacity of inhibiting various bacterial species including S. aureus (28). Pawar and Pal (29) in a study indicated that chloroform and methanol extracts exhibited valuable antimicrobial potency on gram-positive and gram-negative bacteria. The chloroform root extract of $T$. catappa showed antimicrobial activity against Escherichia coli and S. aureus while petroleum root ether extract of T. catappa was devoid of antimicrobial activity. However, the high susceptibility rate of $S$. aureus to this extract may be attributed to the numerous phytochemicals present in the plant.

Modern science and technology have achieved success in drug discovery development. Not with standing, microorganisms in alarming frequency still create resistant pattern to some of the available modern medicines. Chikere et al (30), in a study have reported that the extensive use of broad-spectrum antibiotics has led to the wide spread occurrence of nosocomial infections by multi drug resistant microorganisms. To this extent, the high numbers of $S$. aureus isolated from patients and their resistance structure to majority of the employed modern drugs ascertained the urgency in attention needed to save the world from multi drug resistant microbes by finding alternative effective measures for their eradication. Despite this, the ineffectiveness of some modern medicines is not discriminated as faced on traditional folklores. The continuous use of these ineffective antibiotics however, may favor the emergence of resistant strains of hospital and community acquired pathogens.

\section{Conclusion}

Staphylococcus aureus is a human threatening bacterium that can cause many illnesses in man and animals. OFL, GEN and ERY as recommended first line antibiotics in the management of $S$. aureus infections were not as effective on the isolated $S$. aureus as does by plants extracts. Since T. catappa, M. indica and A. wilkesiana contain several bioactive compounds based on their effective antibacterial potencies, they may serve as sources for novel antibiotics for the treatment of diseases.

\section{Authors' contributions}

MOO and FCA conceived the research idea, designed and wrote the first draft of the manuscript. AOE assisted in literature search. All authors read and approved the final manuscript.

\section{Conflict of interests}

None.

\section{Ethical considerations}

The protocol was confirmed in ethical committee and the authors of this manuscript observed ethical issues (EKSUTH/A67/2016/10/021)

\section{Funding/Support}

This research was financially supported by the authors.

\section{References}

1. Klein EY, Sun L, Smith DL, Laxminarayan R. The changing epidemiology of methicillin-resistant Staphylococcus aureus in the United States: a national observational study. Am J Epidemiol. 2013;177(7):666-74. doi: 10.1093/aje/ kws273.

2. Khan Z, Faisal S, Hasnain S. The continuing threat of methicillin-resistant Staphylococcus aureus-Past Present Future. Journal of Scientific Research. 2010;40(2):31-4.3745.

3. Hammuel C, Jatau ED, Whong CMZ. Prevalence and antibiogram pattern of some nosocomial pathogens isolated from Hospital Environment in Zaria, Nigeria. Aceh Int J Sci Technol. 2014;3(3):131-9. doi: 10.13170/aijst.3.3.1593.

4. Newman MJ. Neonatal intensive care unit: reservoirs of nosocomial pathogens. West Afr J Med. 2002;21(4):310-2.

5. Ahoyo TA, Martin-Odoom A, Bankole HS, Baba-Moussa L, Zonon N, Loko F, et al. Epidemiology and prevention of nosocomial pneumonia associated with Panton-Valentine leukocidin (PVL) producing Staphylococcus aureus in Departmental Hospital Centre of Zou Collines in Benin. Ghana Med J. 2012;46(4):234-40.

6. Harborne JB. Phytochemical Methods. London: Chapman and Hall Ltd; 1973:149-88.

7. Chethana GS, Hari Venkatesh KR, Gopinath SM. Preliminary phytochemical analysis of Clerodendrum inerme. Int Res J Pharm. 2013;4(5):208-9. doi: 10.7897/22308407.04542 .

8. Clinical and Laboratory Standards Institute (CLSI). Performance Standards for Antimicrobial Disk Susceptibility Tests, Approved Standard. 7th ed. Wayne, Pennsylvania: CLSI; 2012

9. Walson D. Principles and Practice of Statistics in Biological Research. 2nd ed. New York: W.H. Freeman; 1989:53.

10. Uwazuoke JC, Aririatu LE. A survey of antibiotic resistant Staphylococcus aureus strains from clinical sources in Owerri. J Appl Sci Environ Manag. 2004;8(1):67-9. doi: 
10.4314/jasem.v8i1.17230.

11. Ndip RN, Ebah LME, Onile BA. Antibiogram of Staphylococcus aureus from clinical syndromes in Ilorin, Nigeria. J Med Lab Sci. 1997;6:24-6.

12. Amadi ES, Ikeagwu IJ, Iroha IR. Antibiotic sensitivity pattern of Staphylococcus aureus in Abakaliki, Nigeria. Pak J Med Sci. 2008;24(2):231-5.

13. Obiazi HAK, Nmorsi OPG, Ekundayo AO, Ukwandu NCD. Prevalence and antibiotic susceptibility pattern of Staphylococcus aureus from clinical isolates grown at 37 and 44oC from Irrua, Nigeria. Afr J Microbiol Res. 2007;1(5):57-60.

14. Akharaiyi FC, Oso BA, Boboye BE, Akinyosoye FA. Bacterial growth inhibition properties and in vivo antioxidant effects of Spondias mombin fruit juice. Roum Arch Microbiol Immunol. 2017;76(1):41-9.

15. Dang-Thic XT, Hoang-Thi KN, Vo TT. Antimicrobial resistance of Staphylococcus aureus isolated from pork in Ho Chi Minh City, Vietnam. Agricultural Science Research Journal. 2014;5(4):62-7.

16. Oguzkaya-Artan M, Baykan Z, Artan C. Nasal carriage of Staphylococcus aureus in healthy preschool children. Jpn J Infect Dis. 2008;61(1):70-2.

17. Onanuga A, Oyi AR, Olayinka BO, Onaolapo JA. Prevalence of community-associated multi-resistant Staphylococcus aureus among healthy women in Abuja, Nigeria. Afr J Biotechnol. 2005;4(9):942-5.

18. Eloff JN. On expressing the antibacterial activity of plant extracts - a small first step in applying scientific knowledge to rural primary health care. S Afr J Sci. 2000;96(3):116-8.

19. Al-Jabri AA. Honey, milk and antibiotics. Afr J Biotechnol. 2005;4(13):1580-7.

20. Oluduro AO, Bakare MK, Omoboye OO, Dada CA, Olatunji CI. Antibacterial effect of extracts of Acalypha wilkesiana on gastrointestinal tract pathogens and bacteria causing skin infections in neonates. Ife J Sci. 2011;13(2):371-80.

21. Akinyemi KO, Oluwa OK, Omonigbehin EO. Antimicrobial activity of crude extracts of three medicinal plants used in south-west Nigerian folk medicine on some food borne bacterial pathogens. Afr J Tradit Complement Altern Med. 2006;3(4):13-22.

22. Onocha PA, Olusanya TOB. Antimicrobial and anthelmintic evaluation of Nigerian Euphorbiaceae plants 3: Acalypha wilkesiana. Afr Scientist. 2010;11(2):85-9.

23. Akpomie OO, Olorungbon S. Antimicrobial screening of Terminalia avicennoides and Acalypha wilkesiana. Afr J Biotechnol. 2011;10(2):180-2. doi: 10.5897/AJB10.457.

24. Awe S, Eme I. Antibacterial activity and phytochemical screening of Acalypha wilkesiana (Copperleaf) leaf extract on some clinical isolates. Res J Pharm Biol Chem Sci. 2014;5(4):1-7.

25. Ncube NS, Afolayan AJ, Okoh AI. Assessment techniques of antimicrobial properties of natural compounds of plant origin: current methods and future trends. Afr J Biotechnol. 2008;7(12):1797-806.

26. Khan AA, Kumar V, Singh BK, Singh R. Evaluation of wound healing property of Terminalia catappa on excision wound models in Wistar rats. Drug Res (Stuttg). 2014;64(5):225-8. doi: 10.1055/s-0033-1357203.

27. Taganna JC, Quanico JP, Perono RM, Amor EC, Rivera WL. Tannin-rich fraction from Terminalia catappa inhibits quorum sensing (QS) in Chromobacterium violaceum and the QS-controlled biofilm maturation and LasA staphylolytic activity in Pseudomonas aeruginosa. J Ethnopharmacol. 2011;134(3):865-71. doi: 10.1016/j. jep.2011.01.028.

28. Akharaiyi FC, Ilori RM, Adesida JA. Antibacterial effect of Terminalia catappa on some selected pathogenic bacteria. Int J Pharm Biomed Res. 2011;2(2):64-7.

29. Pawar SP, Pal SC. Antimicrobial activity of extracts of Terminalia catappa root. Indian J Med Sci. 2002;56(6):2768.

30. Chikere CB, Chikere BO, Omoni VT. Antibiogram of clinical isolates from a hospital in Nigeria. Afr J Biotechnol. 2008;7(24):4359-63. 